\title{
An Implementation of Wireless Medical Image Transmission System on Mobile Devices
}

\author{
SangBock Lee • Taesoo Lee • Gyehwan Jin • \\ Juhyun Hong
}

Received: 2 February 2008 / Accepted: 31 March 2008 /Published online: 5 June 2008

(C) The Author(s) 2008

\begin{abstract}
The advanced technology of computing system was followed by the rapid improvement of medical instrumentation and patient record management system. The typical examples are hospital information system (HIS) and picture archiving and communication system (PACS), which computerized the management procedure of medical records and images in hospital. Because these systems were built and used in hospitals, doctors out of hospital have problems to access them immediately on emergent cases. To solve these problems, this paper addressed the realization of system that could transmit the images acquired by medical imaging systems in hospital to the remote doctors' handheld PDA's using CDMA cellular phone network. The system consists of server and PDA. The server was developed to manage the accounts of doctors and patients and allocate the patient images to each doctor. The PDA was developed to display patient images through remote server connection. To authenticate the personal user, remote data access (RDA) method was used in PDA accessing the server database and file transfer protocol
\end{abstract}

\author{
S. Lee $(\bowtie) \cdot$ G. Jin \\ Department of Radiology, Nambu University, \\ 864-1, Wolgye-dong, Gwangsan-gu, Gwangju, \\ Gwangju-city 506-706, South Korea \\ e-mail: sblee@nambu.ac.kr \\ G. Jin \\ e-mail: ghjin@nambu.ac.kr \\ T. Lee $\cdot$ J. Hong \\ Department of Biomedical Engineering, College of Medicine, \\ Chungbuk National University, \\ Cheongju, South Korea \\ T. Lee \\ e-mail: tslee@chungbuk.ac.kr \\ J. Hong \\ e-mail: brood75@chungbuk.ac.kr
}

(FTP) was used to download patient images from the remove server. In laboratory experiments, it was calculated to take ninety seconds to transmit thirty images with $832 \times 488$ resolution and 24 bit depth and $0.37 \mathrm{Mb}$ size. This result showed that the developed system has no problems for remote doctors to receive and review the patient images immediately on emergent cases.

Keywords Personal digital assistant (PDA) . Medical image $\cdot$ CDMA $\cdot$ Wireless transmission

\section{Introduction}

The aging of society and changes in food-related lifestyle are increasing intractable diseases such as hypertension, diabetes and cancers, the treatment of which costs a lot of money and time. This trend is naturally increasing medical expenses. Korea has already entered into an aged society, and is expected to be a super-aged society in 2019 where the aged will occupy around $14 \%$ of the population [1]. The aging of society means the rise of medical expenses, and this will bring a heavy burden to national economy. Demand for medical services will increase and consequently medical costs will also rise. To solve these problems, researches are being made recently on medical supports using electronic computing technologies such as ubiquitous (at any time and in any place) healthcare. In addition, although hospitals should give priority to patient treatment, they still need to enhance the efficiency of management, and for this, they should introduce and apply technologies that enable advanced medical service and management.

With such changes in medical service environment and thanks to the development of embedded system technologies and communication infrastructure, researches are being 
made actively on medical systems based on portable computing technologies and body activity monitoring $[2,3,4]$. The advance of embedded systems and communication devices makes it more flexible to acquire, distribute and manage information through medical equipment, and improves existing hospital information systems (HIS) based on text information so that they can provide more convenient and systematic functions to direct users such as doctors and nurses as well as indirect users such as patients. Among the hospital information systems, representative systems related to patient mages include picture archiving and communication system (PACS) that can acquire, store and manage images, digital imaging and communication system in medicine (DICOM), and radiology information system (RIS) [5]. For example, PACS [6, 7], which has been unprofitable because of the continuous rise of the price of supplies and management expenses in patient image management systems using films, costly equipment and personnel and low speed, is now less costly to introduce thanks to cheaper hardware and abundant supply of operators. In addition, Web-based patient image management and inquiry systems have been being introduced widely with the support of the Internet and highspeed communication systems [8, 9]. Recently, in the USA, the amount of daily medical expenses is around $\$ 2$ billion or $13 \%$ of the gross national product. The amount has been increasing steadily, and accordingly millions of Americans cannot access medical services and many hospitals in urban and rural areas are closing their business due to degenerated quality of medical services and financial difficulties. Accordingly, one of American companies' biggest concerns is the control of medical expenses, and the state and federal governments are struggling to manage the claims of medical expenses. One of methods considered to overcome these problems is the introduction of telemedicine. Telemedicine is a system that supports consultation, medical specialist education, patient information management and transmission, electronic billing and processing of medical expenses, and electronic inventory management system from a remote place. The introduction of the system is expected to reduce medical expenses by over $\$ 36$ billion a year. However, such a telemedicine and image management $\&$ inquiry system is operable only in places with required equipment, and thus its operation is highly restricted. For example, it is very hard to get medical services for those in a remote mountain village or an island without medical facilities. This limitation may worsen diseases and lead to the loss of lives in case simple treatment and diagnosis by a specialist could have saved the patients. To prevent this problem, early diagnosis of patients by a specialist is a very important element. What is more, in many cases, patient images taken unexpectedly in hospital need to be diagnosed immediately by a medical specialist, but with current available medical systems the specialist has to visit the hospital or the image has to be delivered to the doctor. Thus, in the USA, there are radiologists working at night who read emergent patients' images in other hospitals through teleradiology. To ease the situation, patient images can be viewed through a desktop system connected to the Internet, but this method is not useable to doctors in traveling. For immediate diagnosis by a doctor at any time and in any place, we need a system that can send urgent patient images, and this demands a technology that can overcome the limitation of time and space using only portable equipment.

The most popular portable system, namely, PDA (personal digital assistant) is inferior to desktops and laptops in performance, but considering that it is portable we can say that it has a very large computing capacity. For example, hx2190 PDA of HP can operate at a speed of $312 \mathrm{MHz}$ using PXA270 processor of Intel, and hv kh1000 model of Hanvit IT Co., Ltd. can compute at $400 \mathrm{MHz}$ using S3C2440A processor of Samsung Electronics. This computing capacity enables not only general operations but also multitasking using a multi-processing operating system. Currently, operating systems such as Linux and Windows CE are commonly used. Although PDA has a problem in high-quality image processing, it shows very high performance in processing ordinary images [10]. Due to its characteristics, PDA is vulnerable in network communication. Not only the power consumption problem but also its problem in communication should be solved urgently to guarantee mobility in any place. Currently used communication methods embedded in PDA or used in the form of an externally connected device include infrared data access (IRDA), Bluetooth, wireless local area network (WLAN) and code division multiple access (CDMA). IRDA is a low-speed communication method for a very short distance. Bluetooth is for a short-range communication at speed of 1 Mbps within an office-size space, and is used commonly in simple communication between devices. Like Bluetooth, WLAN also implements wireless communication in spaces such as offices and can attain a speed as fast as local area network (LAN). In order to use WLAN, however, there should be an access point (AP) nearby and AP operates in connection to routers or hubs used in wired LAN, and therefore, WLAN cannot be used in a place without such facilities. There are WLAN-based mobile DICOM image inquiry systems, but they are used limitedly only in places with related facilities [10]. CDMA uses existing CDMA communication networks built for voice communication and EV-DO (evolution-data optimized) modem, which is a wireless data communication technology implemented on chips developed by Qualcom and adopted as a standard by 3GPP2 (3rd Generation Partnership Project2) and ITU (International Telecommunication 
Union). Because the EV-DO modem was developed for data communication, it does not support voice communication and is used as a terminal exclusively for EV-DO networks.

Currently, CDMA-based mobile phone networks have been built widely and thus we can make a voice call using a mobile phone in any place. Wireless data transmission through CDMA as a voice communication network is drawing people's attention as a next-generation wireless transmission system. The present study used a CDMA communication network in order to allow doctors to access patient images through a portable PDA at any time and in any place. There have been many researches on systems for patient image inquiry or chart input and inquiry through PDA and portable terminals in WLAN or offline environment [11-15]. The image transmission method used in this study was implemented with an EV-DO modem used in CDMA networks. In a portable device, the user was authenticated with database information stored in the server using the database replication method. Database synchronization methods between a portable device and the database server are largely divided into MR (merge and replication) and RDA (remote data access), and we used RDA. MR synchronizes database through the merge and replication functions of database management system (DBMS). This method is used mainly for synchronization at a specific point of time rather than real-time synchronization with the server. For example, in case one works outside and has to access data inside, he fetches the data of the internal database through MR and synchronizes the data when the contents change or periodically. This method can save communication expenses because network connection and data update are made only when they are necessary. However, because we cannot predict when the contents of the database will change, we have to carry old data. Because RDA accesses the SQL server directly whenever necessary, it should maintain the connection continuously and this increases communication load and expenses compared to MR, but its speed is faster than MR.

This study constructed a server system that classifies image data collected from patient image acquisition equipment in hospitals by doctor/patient, classifies and stores patient images according to doctor in charge, and provides files in response to a request from PDA on CDMA networks. In addition, a processing system was implemented in client PDA that can download and view patient images at any time and in any place through CDMA communication.

In the next chapter "Materials and Methods," we will explain the composition of system, the hardware and implementation methods, and in 'Results and Discussion,' we will discuss the implemented system and programs. Lastly, in 'Conclusions,' we will review the implemented system and suggest tasks for future research.

\section{Materials and methods}

The system in this study is largely divided into the server system and PDA, and is composed as in Fig. 1.

The server system classifies image files received from image acquisition equipment by patient and stores them so that it can provide them to PDA at any time. Hereinafter the server will be called IRS (image repository server). IRS is an IBM-compatible workstation using Microsoft Windows as its operating system. IRS manages connection to PDA and performs registration, deletion and update of information on doctors, patients and images. It also authenticates

Fig. 1 Construction of total System

Medical Telemetry

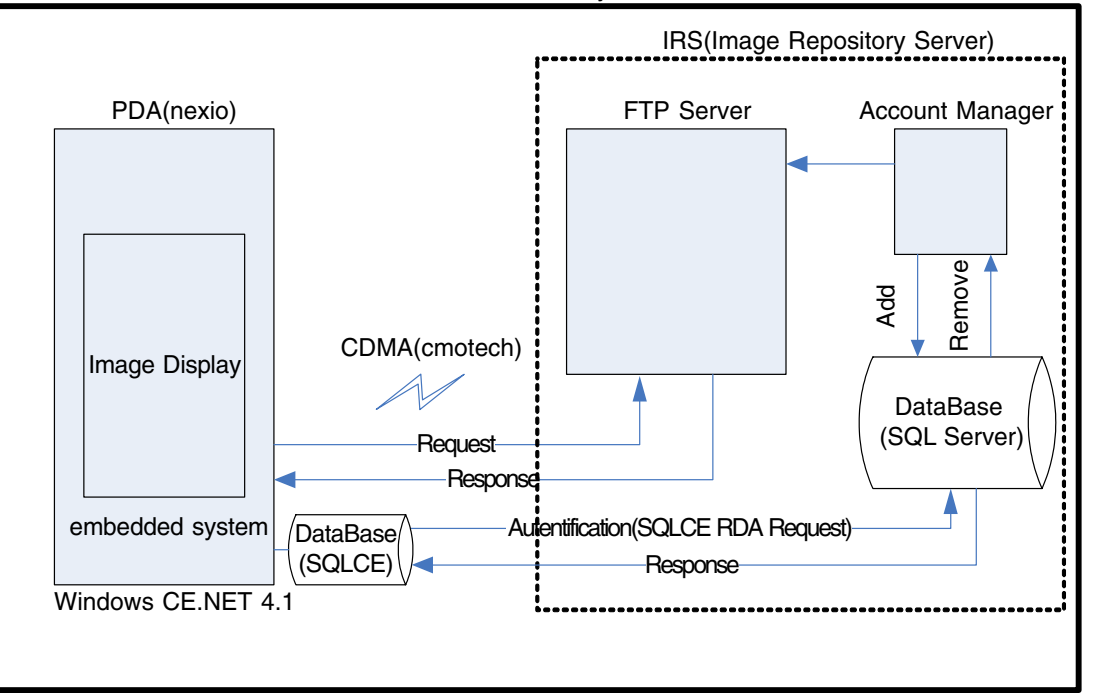


Table 1 IRS database schema

\begin{tabular}{lc} 
Information & \\
\hline Doctor information & doc_info(d_number, d_id, d_license, \\
& d_pass, d_name, d_etc) \\
Patient information & pat_info(p_number, p_name, \\
& p_licnum, p_doc, p_sickname $)$ \\
Images information & img_info(i_pat,i_date,i_num,i_etc) \\
\hline
\end{tabular}

users who access the system and plays the role of a file server.

In IRS, PDA connection processing uses FTP (file transfer protocol), and user authentication uses a databasebased authentication process. The IRS system was developed using Microsoft Visual Studio.NET 2005 C\#, and DBMS used was Microsoft SQL Server 2000 SP4. In Fig. 1, IRS can be divided into two components: FTP Server and Account Manager. Account Manager generates new accounts and manages images by patient, and FTP Server is in charge of clients' connection. When a new account is registered, Account Manager generates an account and repository necessary for FTP. User accounts generated by Account Manager are replicated and used in a portable system at need. The schema of the major database used in the system is as follows (Table 1).

In IRS, image files are handled separately from the database. The database maintains information on doctors, patients and image serial numbers, and image files generated by imaging equipment in hospitals exist in the form as in the figure below. Therefore, the database does not contain image files but stores only data on doctors, patients and image numbers (Table 2).

For example, an image file for Patient 201001 will be stored in FTP_root/doctor/201001, which is a storage place for Patient 201001 under a doctor whose registration number is 101034. Following the rule, the user accessing through a portable terminal is connected to the place corresponding to the user ID in the server, and the user can download images under his management. The PDA connected to IRS was Nexio XP30 of Samsung Electronics. The following table shows the brief specifications of Nexio XP30 (Table 3).

As shown above, XP30 has a high-resolution display $(640 \times 480)$ different from other portable systems, so it is efficient in displaying and processing image data. What is

Table 2 Formation of data file

101034_201001_20060102_001.jpg

\begin{tabular}{ll}
\hline$[101034]$ & Doctor No(1:ID_No,01:Date,034:Serial No.) \\
{$[201001]$} & Patient No(2:ID_No,01:Date,001:Serial No.) \\
{$[20060102]$} & Exposure Date(Year/Month/Day) \\
{$[001]$} & Serial No. \\
\hline
\end{tabular}

Table 3 Principal specification of Nexio XP30

\begin{tabular}{ll}
\hline Specifications & \\
\hline Model & Nexio XP30 \\
Display & 5inch Wide VGA $(800 \times 480)$ TFT LCD, 64K color \\
O/S & Windows CE.NET 4.1 \\
CPU & Intel PXA255 400 MHz \\
Memory & Flash ROM 64 MB(NAND형), SDRAM 128 MB \\
Expansion & Compact Flash slot, standard USB port(1.1) \\
Battery & Rechargeable Li-Polymer 1500 mAH \\
Etc. & $802.11 \mathrm{~b}$, Wi-Fi certified, 11 Mbps \\
Weight & $154 \times 91 \times 13.4(\mathrm{~mm}), 240 \mathrm{~g}$ \\
\hline
\end{tabular}

more, while most PDAs function as the client of a USB port and therefore they cannot use external devices using a USB port, XP30 provides the USB host function so that it can use USB-type peripheral devices such as memory stick and external LAN card. Based on the USB host function, we built CDMA communication connection to IRS using CCU-550 of Cmotech, an external USB-type EV-DO modem. Despite the advantages, however, XP30 uses Windows CE.NET 4.1 as its operating system, which is older than Windows CE Mobile 5.0. For this reason, there are many restrictions in implementing applications, which come from the insufficient support of SDK for serial control, Web-based applications, etc. The communication of the portable system is wireless communication using a

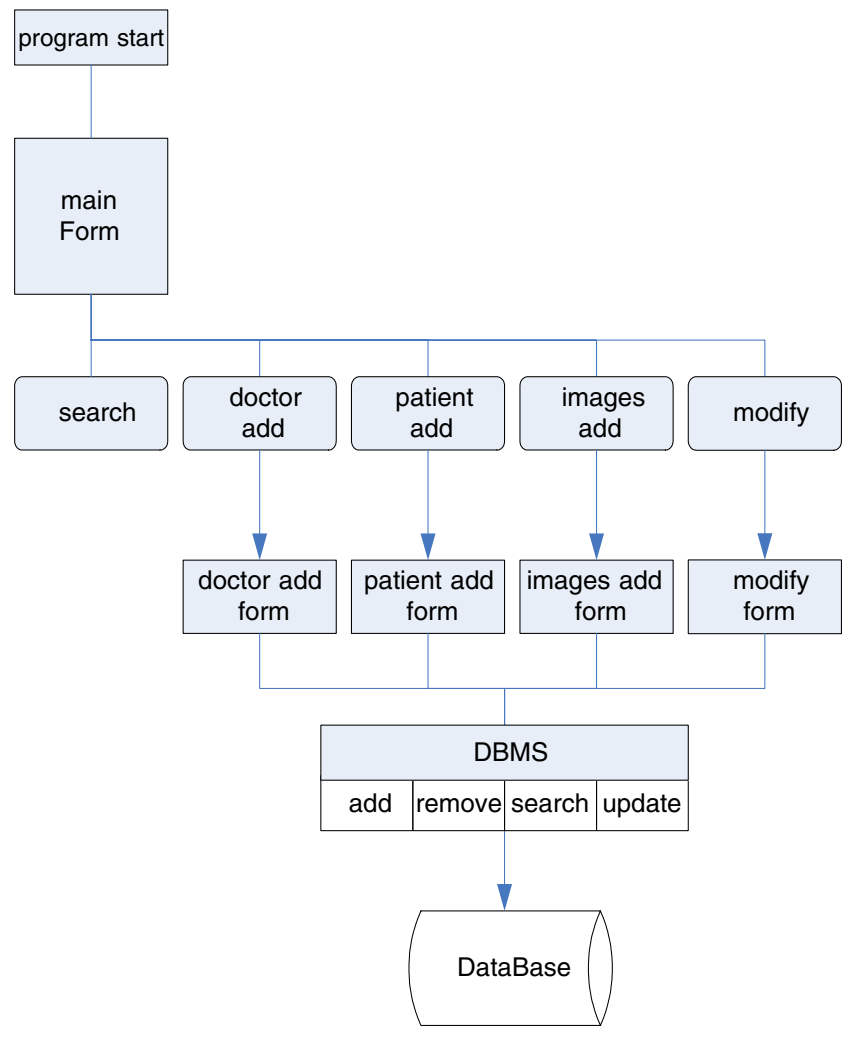

Fig. 2 IRS Flowchart 
Fig. 3 Connection between PDA

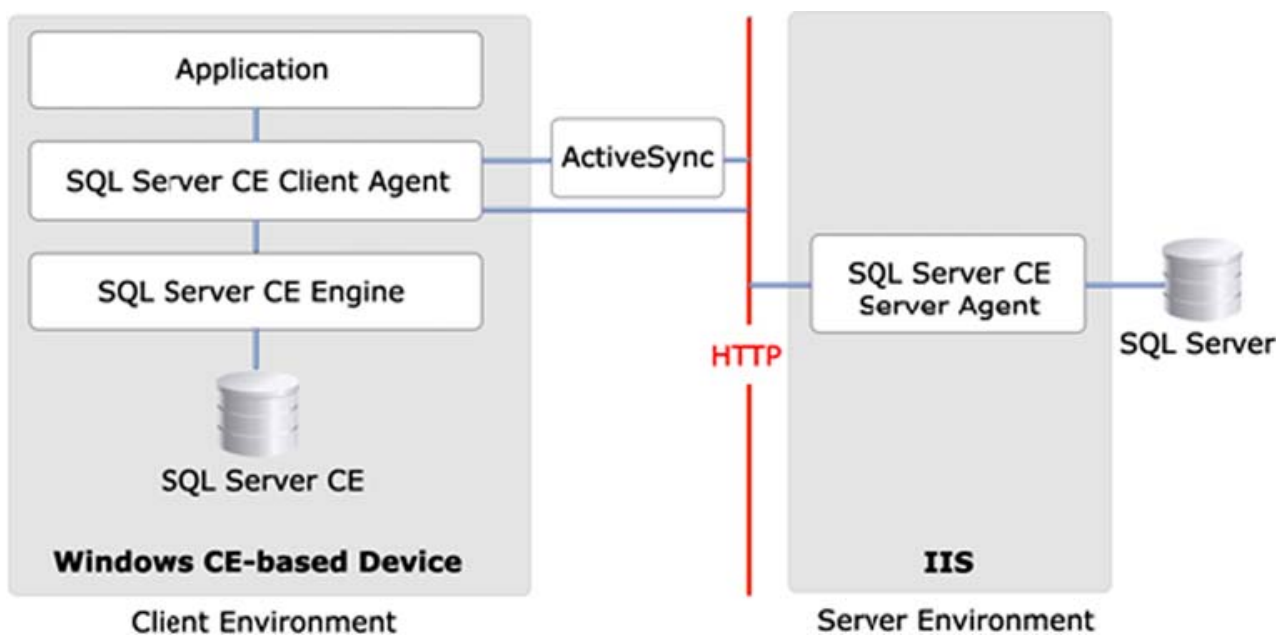

CDMA modem. CCU-550 used in this study adopts the frequency band of $800 \mathrm{MHz}$, and can transmit data at a rate of up to $2.4 \mathrm{Mbps}$. CCU-550 is a USB-type external CDMA modem and is usable in Nexio XP30. For the use of the modem, first, the driver should be registered in the system in order to use the USB port in XP30 and the device should be recognized using the port recognition program provided by the manufacturer. After the device is recognized, modem registration and connection process are performed and then the modem can be used like WLAN.

Using the components described above, this study implemented a wireless image transmission system based on a CDMA network. The entire system is composed of IRS running on a desktop and the portable system running on PDA. The flow diagram of the IRS program is as follows (Fig. 2).

In the IRS system, remote connection process uses FTP, and when an account for a doctor or a patient is generated, a repository is created according to the defined rules in the designated root directory of FTP. The Account Manager program shows the main menu on [mainFrm] and waits for the user's input. The menu includes account generation, deletion and change.

Second, the portable system uses a CDMA modem as a connector, and files are received through FTP. In order to view image information in the portable system, the user needs to be authenticated by the authentication system based on ID and password, and for this the PDA needs to access the server database. The server uses Microsoft SQL 2000 SP4 as its DBMS, and a SQLCE 2.0 database system was built for database access through PDA. The two software architectures are composed and operated as in the figure below (Fig. 3).

In the figure, the server side has Internet information server (IIS) for Web services, which performs synchronization with the SQL server through Server Agent implemented with Internet server application programming interface (ISAPI). SQLCE delivers Client Agent's request to the SQL server through SQL Server CE Server Agent (SSCESA20.d1l).
Fig. 4 Diagram of connected cons
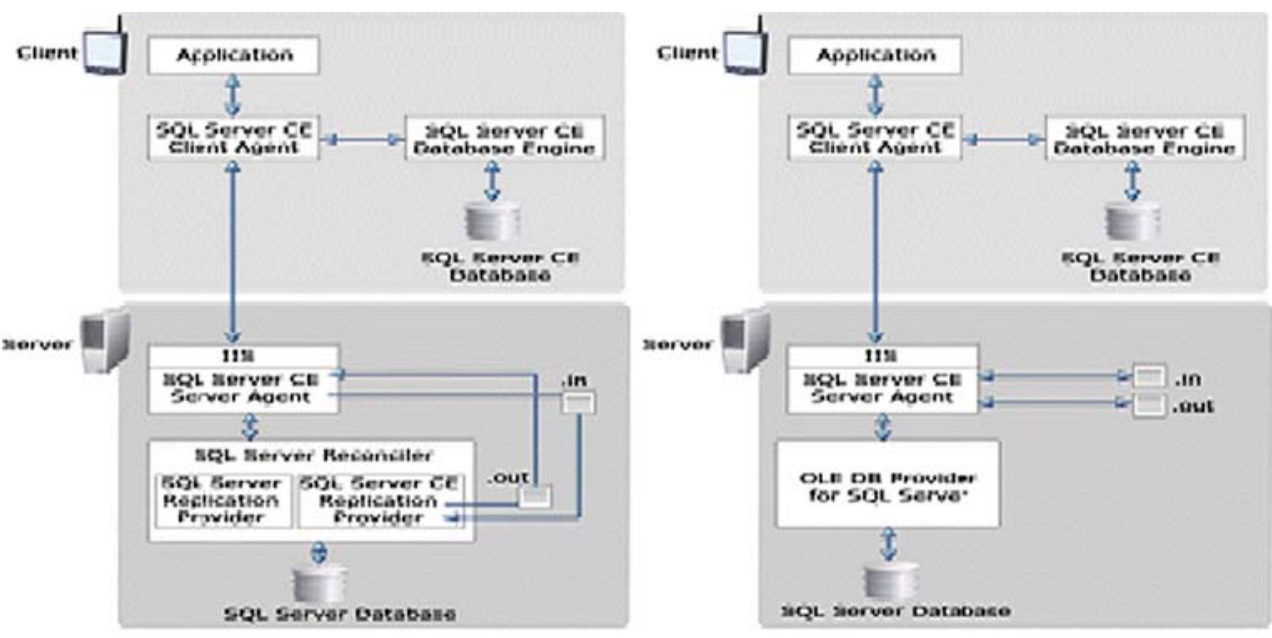
Fig. 5 PDA flowchart

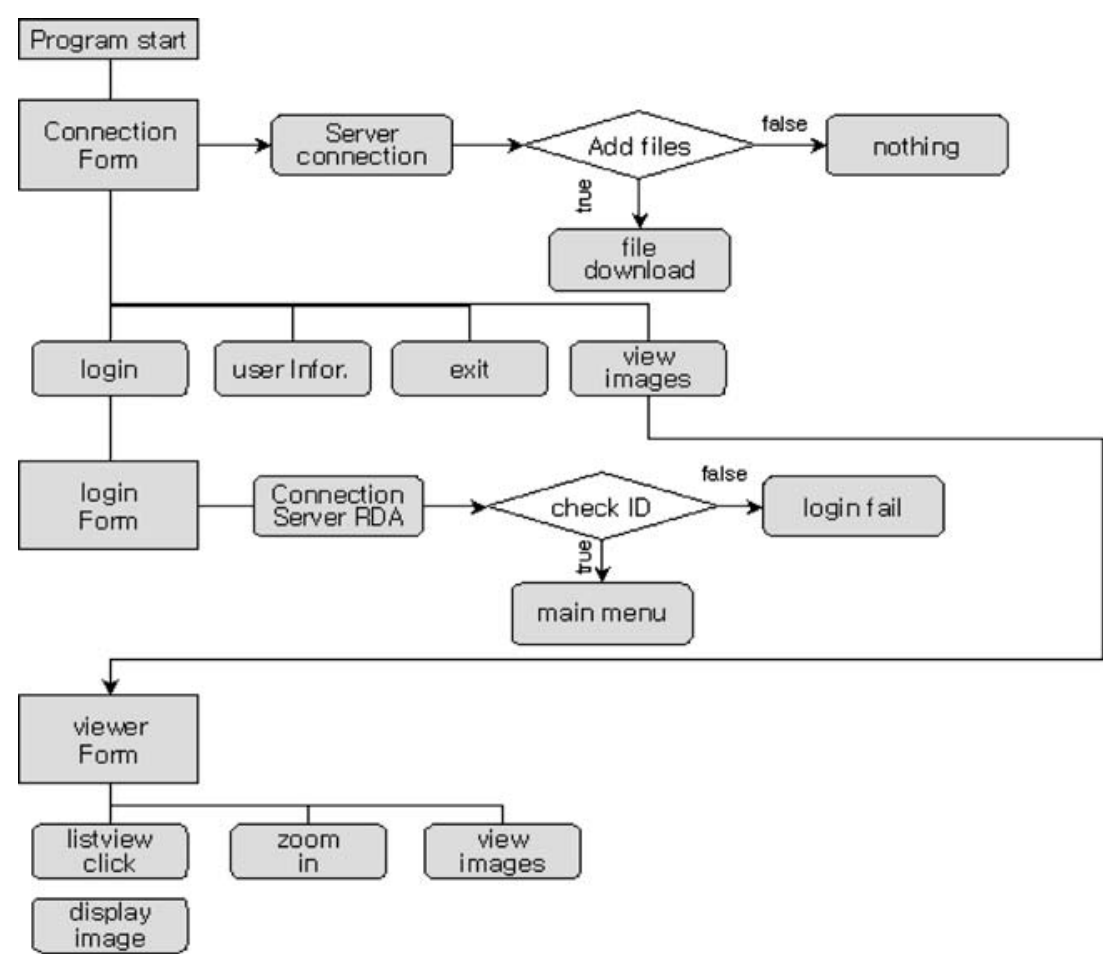

Depending on use, the server interacts with a client through either RDA or MR. The figure below shows RDA and MR (Fig. 4).

MR synchronizes with SQLCE using the merge and replication function of DBMS. This method replicates the database in the server and stores it in the SQLCE database of PDA. In case of SQLCE database, a database is a physical file. RDA carries out desired tasks by accessing a remote SQL server through network connection. Connection to a server by RDA should always be activated for database access, and this may increase communication load and cost.

In order to access patient images in the server through PDA at any time and in any place and to allow only approved users to access data, we implemented a database authentication system through network connection using the RDA method. In addition, authenticated users can navigate, enlarge or reduce downloaded patient images, and if necessary, they can connect to the server system and download new images. The operating system of the Nexio XP30 model is Windows CE.NET 4.1. Thus, the development environment is not supported in Windows Visual Studio 2005, and development can be done with a development tool like Embedded Visual Studio Version 4.0 or higher and Windows Visual Studio.NET 2003. Therefore, we developed the PDA client using Windows Visual Studio. NET 2003 C\#. The following block diagram shows the main modules and flow of the PDA system (Fig. 5).

PDA performs functions such as database synchronization through RDA, image file download through FTP, and display of download images. This part gives a detailed explanation about the implementation of FTP. FTP is the most efficient file transmission/reception protocol among Internet services. Therefore, we used the FTP protocol for image transmission. In addition, some of FTP client functions were implemented in PDA and used in server directory listing and file download.

FTP functions are largely divided into initialization, connection establishment, command transmission, command results reception and data reception. FTP supports active/ passive mode and binary/ASCII mode, and was implemented in multiple threads. The program was implanted in $100 \%$ managed code. Here, managed code means code that can be

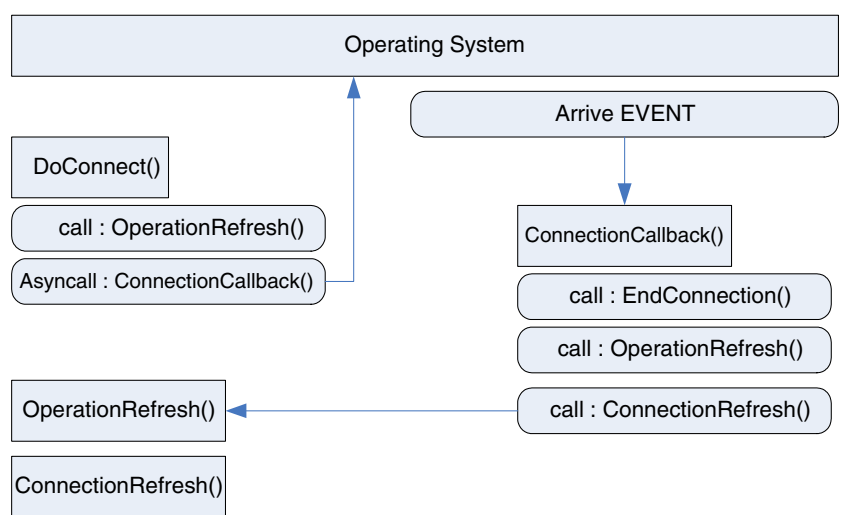

Fig. 6 FTP connection procedure using asynchronous callback 
Fig. 7 The implemented system

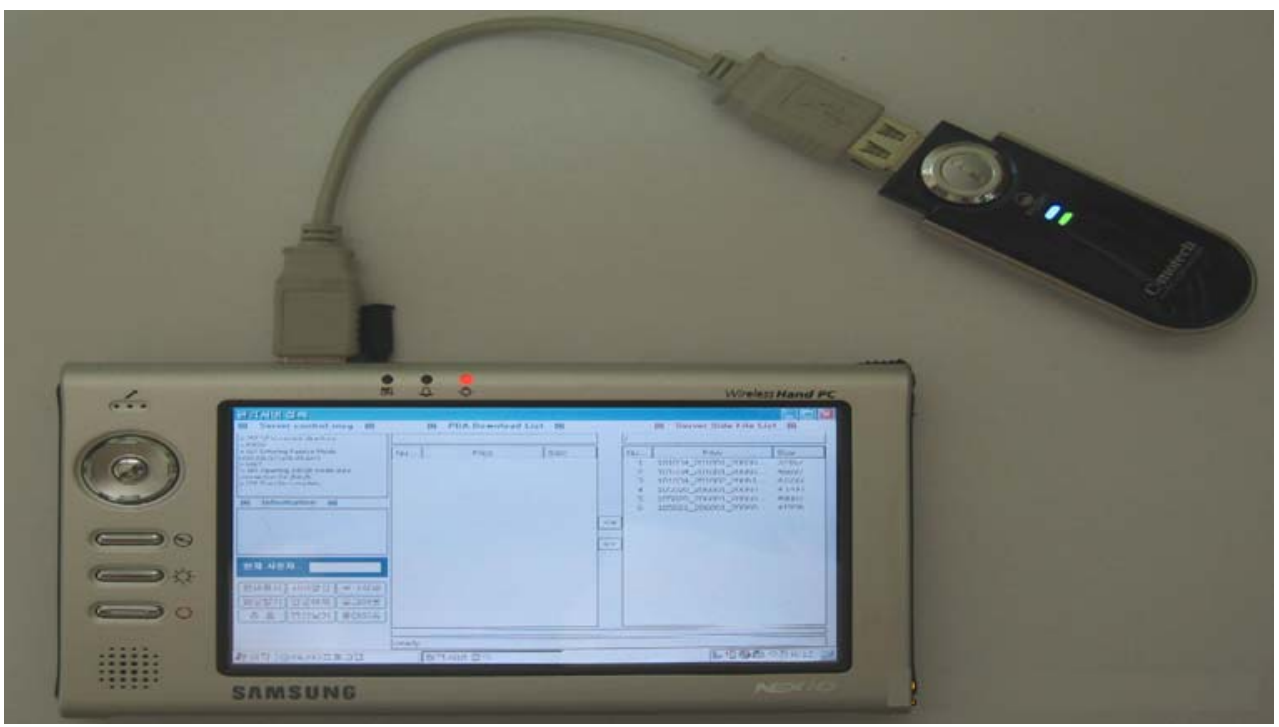

recognized and executed by.NET Framework and, on the contrary, unmanaged code means code that Windows can execute directly without the involvement of.NET Framework. Because FTP commands are executed through synchronization with the server, a reply may come immediately or in a while after a command has been sent. Thus, each operation was implemented in the asynchronous callback method. Because asynchronous callback generates and executes a new work thread separately from the main thread, it is executed independently. In addition, due to the characteristics of callback, if a call function is registered in asynchronous callback, the registered function is called in response to an event in the operating system. The figure below shows the FTP connection process using the asynchronous callback method (Fig. 6).

\section{Results and discussion}

This study implemented a system based on CDMA network, with which a doctor can view patient images at any time and in any place. In order to verify users' access right to the system, we used a database as well as RDA for database synchronization. The implemented system is as in the figure below (Fig. 7).

As in the figure, Nexio XP30 uses a USB-type external device for CDMA communication. In order to use the image management system in the main menu of the program, the user should log on and the figure below is the logon screen through CDMA (Fig. 8).

If a user (a doctor) logs on the system database, the screen, as in the figure below, shows the list of accessible

Fig. 8 The login screen through CDMA

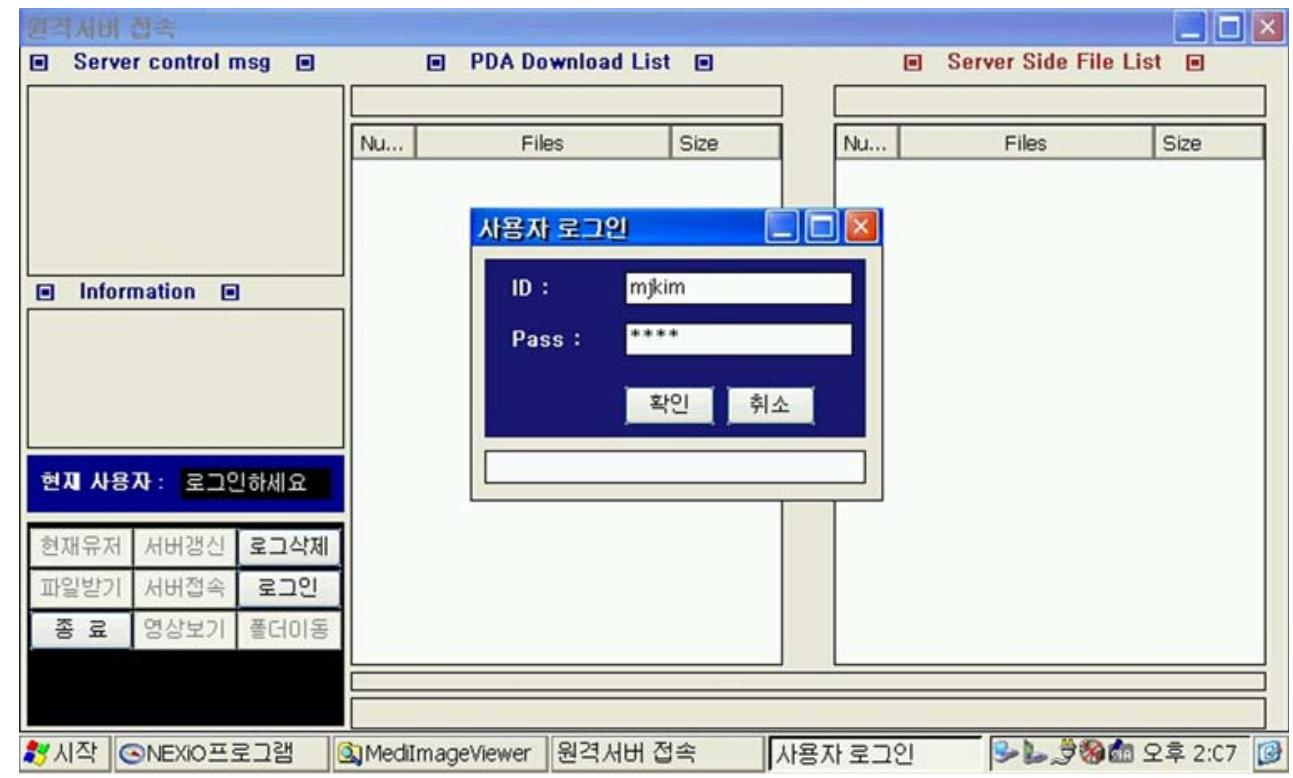


Fig. 9 Patients' image files displays

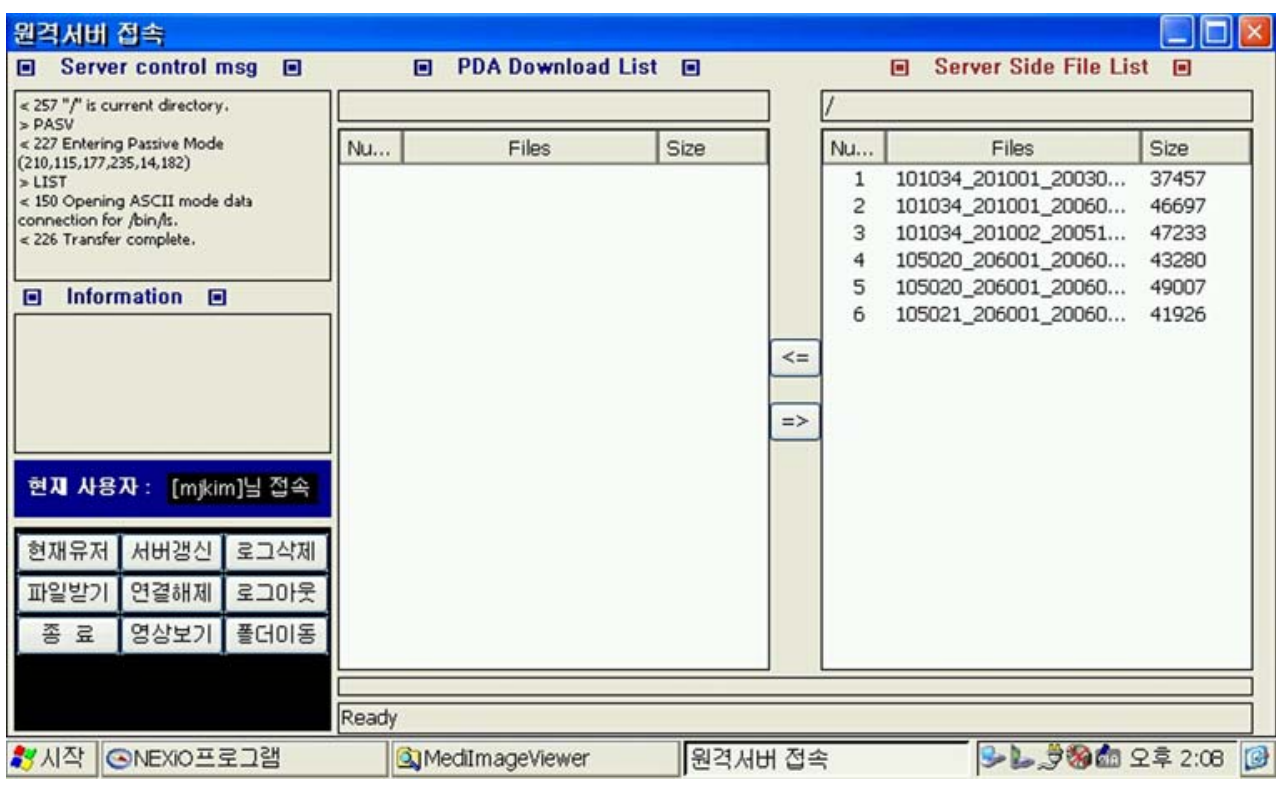

patient image files in the server repository corresponding to the doctor's account. If desired files are selected, those selected are marked as in the figure below, and they can be downloaded and viewed (Figs. 9 and 10).

MR and CR images downloaded from the server can be navigated, enlarged and reduced as in Figs. 11 and 12 through the viewer implemented in PDA, and be presented as in the figure below.

In the Table 4, described files transmission time in this paper.

\section{Conclusions}

This study implemented an image transmission system based on CDMA connection so that users can view images in the server at any time and in any place. Telemedical services based on medical image transmission using PDA can be applied to places where mobility is required, and PDA can be an ideal means for point-of-care. The use of PDA enables the fast and accurate real-time transmission of digital medical images regardless of time and place, and consequently, reduces medical accidents, enhances the productivity and efficiency of medical practitioners' works, and improves the quality of medical services. What is more, it can heighten patients' satisfaction through processing their demands promptly and providing more and better medial services.

The system is composed of a server system for user management and image management and a client system for receiving and viewing images in a portable terminal,
Fig. 10 The user select files can be downloaded and viewed

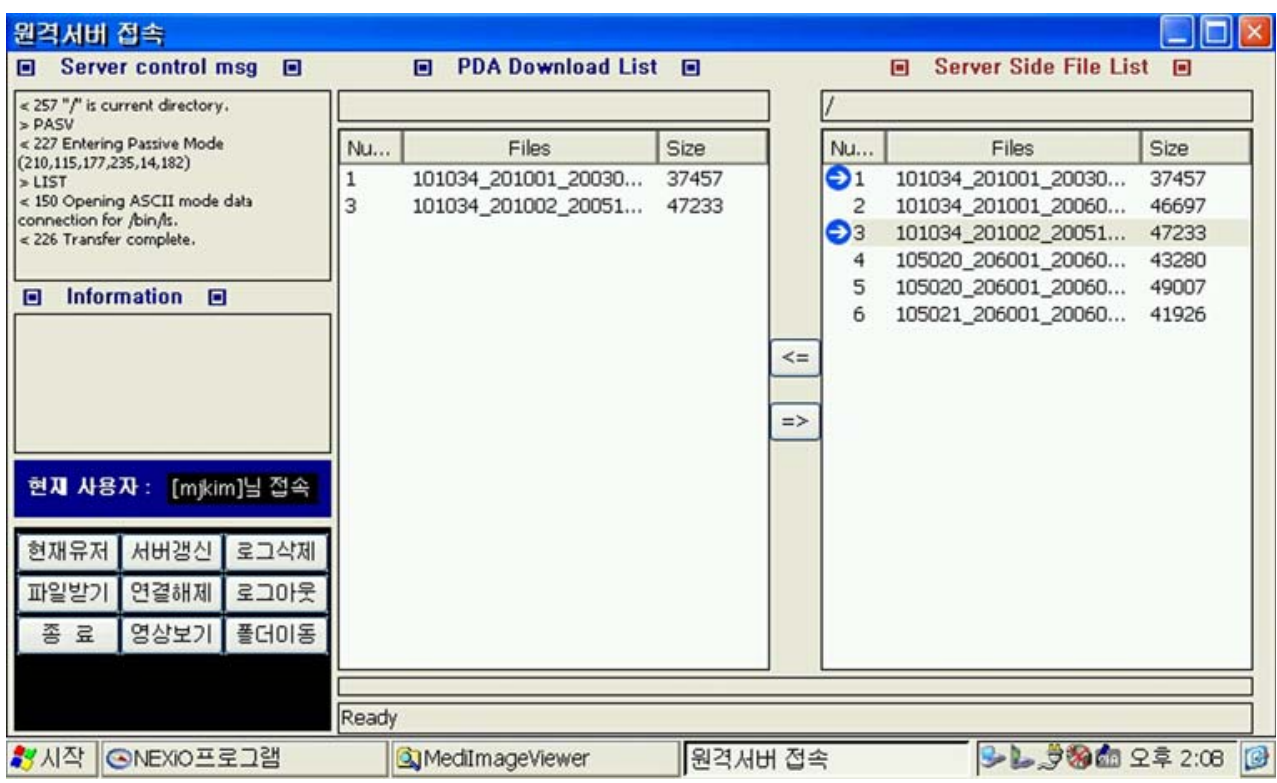


Fig. 11 MR images downloaded from the server

Fig. 12 CR images downloaded from the server
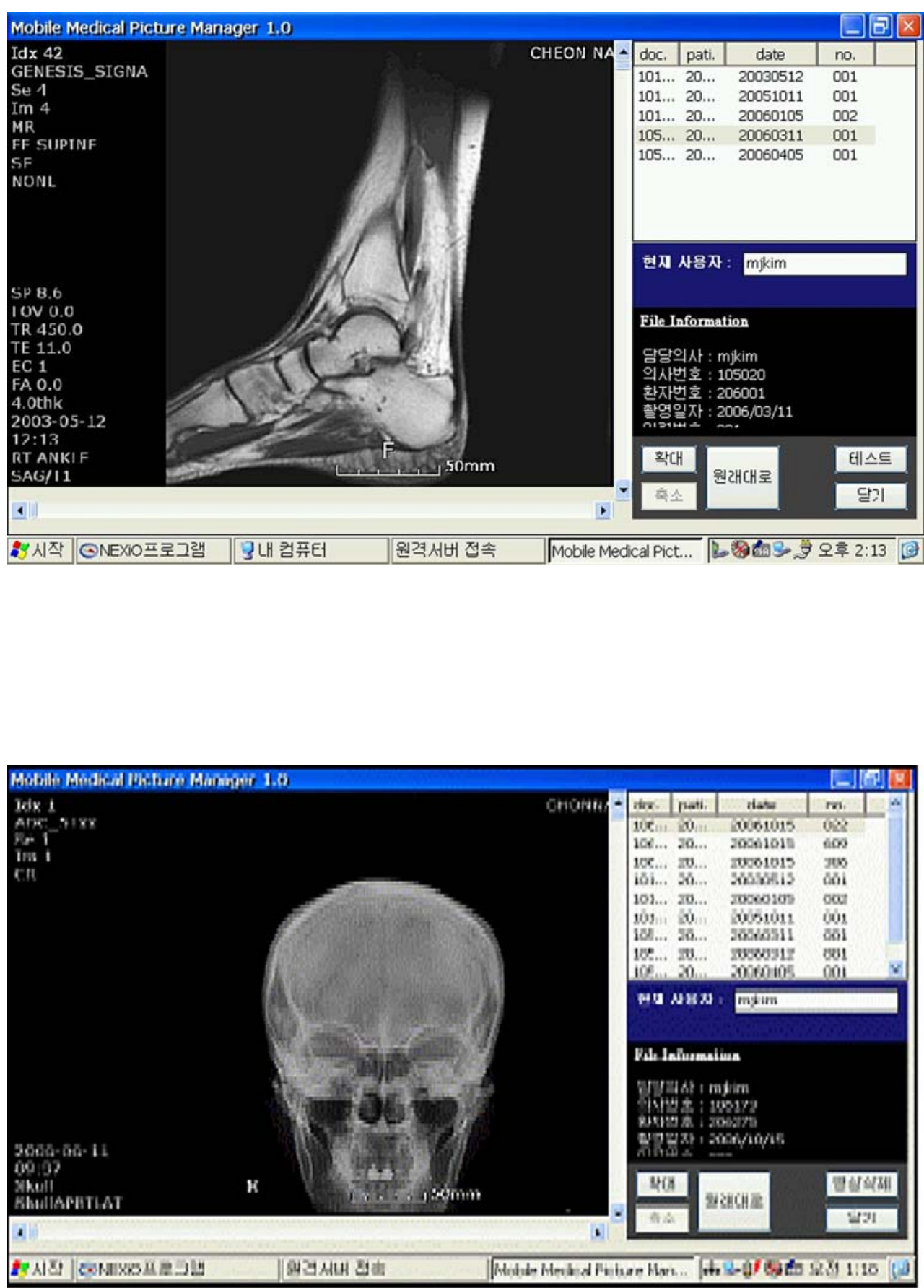

Table 4 Downloading time according to file format

\begin{tabular}{|c|c|c|c|c|}
\hline File format & Pixel & Size (M) & Slice & Transmission time (s) \\
\hline $\mathrm{CT}$ & $512 \times 512 \times 12$ & 0.4 & 30 & 62.1 \\
\hline MRI & $256 \times 256 \times 12$ & 0.1 & 30 & 15.5 \\
\hline Converted into JPG & $832 \times 488 \times 24$ & 1.2 & 30 & 186.3 \\
\hline
\end{tabular}


which was implemented with Nexio XP30 PDA. In general, PDA has a memory of 32 64 MB, which is not sufficient for large-volume data. Thus, each DICOM image is converted into a jpg file, a lossy compression image format, to be provided to PDA. The image conversion may result in damage to precise medical images. However, Nexio XP30 can display an $800 \times 480$ image in $64 \mathrm{k}$ colors, and it has been found by previous research that damage to areas invisible due to the limitation of human sight in recognizable resolution does not cause any problem. Because an image converted to a jpg file was reduced considerably in size, it also reduced the load of transmission and storage on PDA substantially. The medical image transmission implemented in this study attains very high portability and functionality in that it allows doctors to read and diagnose patient images at any time and in any place.

Images taken at local medical institutions without radiologists can be transmitted through communication networks to a radiologist to be read, and therefore, radiology diagnosis can be made promptly at a remote place. Although there are still many problems and difficulties in commercializing and popularizing wireless teleradiology medical systems, it is obvious that, with progress in informatization and civilization of society, there will be increasing demands for such systems and they will be introduced, spread and settled through research and cooperation among the government, research institutions and medial institutions.

The human body produces various types of visible biological information as well as physical information like temperature, movement, blood pressure, ECG, EEG and $\mathrm{SpO} 2$. The medical image transmission system will provide the base for future systems, with which doctors monitor and read various biosignals at any time and in any place in the form of a separate or integrated unit, and research will be continued in this direction. A short-term goal is offline implementation due to limitations in system hardware and software, and furthermore, online observation systems will be developed. In building an online system, there will be difficulties in real-time receiving and processing of biosignals. However, it may be possible for doctors to receive required one among patients' biosignals selectively using a portable terminal and to make more accurate diagnosis at any time and in any place.

Acknowledgment This study was supported by a grant from the Korea Health 21 R\&D Project, Ministry of Health \& Welfare, Republic of Korea (Grant No. A040032).
Open Access This article is distributed under the terms of the Creative Commons Attribution Noncommercial License which permits any noncommercial use, distribution, and reproduction in any medium, provided the original author(s) and source are credited.

\section{References}

1. Hyung-Su, K., The aged problem of aging society. The Korean Association of Humanities and Social Sciences. 23:480-99, 2000.

2. Raskovic, D., Martin, T., and Jovanov, E., Mobile monitoring application for wearable computing. Journal of the Computer. 47:4495-504, 2004.

3. Istepanian, R. S. H., Jovanov, E., and Zhang, Y. T., Guest editorial introduction to the special section on M-health: Beyond seamless mobility and global wireless health-care connectivity. IEEE Trans. Inf. Technol. Biomed. 8:4405-414, 2004.

4. Hong, J. H., Kim, N. J., Cha, E. J., and Lee, T. S., Development brief of a body area network for ubiquitous healthcare: An introduction to ubiquitous biomedical systems development center. J. Biomed. Eng. Res. 26:5331-335, 2005.

5. Kim, D. S., and Park, H. Y., A study of information situation \& growth strategy in a Korea large hospital. Information Policies. 11:413-29, 2004.

6. Kang, D. S., Teleradiology: Wooljin Medical Center and Kyungbook National University Hospital. Journal of The Korean Society of PACS. 1:49-52, 1995.

7. Choi, S. W., Interview: Internet teleradiology system. Journal of The Korean Society of PACS. 2:29-33, 1996.

8. Kwun, K. B., and Kim, I. K., Web-based medical information system supporting DICOM specification. Journal of The Korea Information Science Society. 7:4317-323, 2001.

9. Kwun, K. B., and Kim, I. K., P2P DICOM system using multiagent systems communicating with XML encoded ACL. Journal of The Korea Information Science Society. 8:5598-606, 2002.

10. Cho, J., Kim, D. S., Shin, D. K., and Kim, D. Y., A study on the development of mobile emergency medical image management system using personal digital assistance. Journal of The Korean Society of PACS. 9:17-22, 2003.

11. Lin, Y., Jan, I., Ko, P., Chen, Y., Wong, J., and Jan, G. A., Wireless PDA based physiological monitoring system for patient transport. IEEE Trans. Inf. Technol. Biomed. 8:4439-447, 2004 (Dec).

12. Woodward, B., Istepaniam, R. S. H., and Richards, C. I., Design of a telemedicine system using a mobile telephone. IEEE Trans. Inf. Technol. Biomed. 5:13-15, 2001.

13. Fischer, S., Stewart, T. E., Mehta, S., Wax, R., and Lapinsky, S. E., Handheld computing in medicine. J. Am. Med. Inform. Assoc. 10:2139-149, 2003.

14. Maglogiannis, I., Apostolopoulos, N., and Tsoukias, P., Designing and implementing an electronic health record for personal digital assistants (PDA's). International Journal for Quality of Life Research. 2:163-67, 2004.

15. Rosser, J. C. Jr., Bell, R. L., Harnett, B., Rodas, E., Murayama, M., and Merrell, R., Use of mobile low bandwidth telemedical techniques for extreme telemedicine applications. J. Am. Coll. Surgeons. 189:398-404, 1999. 